\title{
MISURA DEI LENTI MOVIMENTI VERTICALI DELLA SUPERFICIE TERRESTRE (*)
}

\author{
Silvio Polli
}

1. Premesse. - Consideriamo i movimenti verticali della superficie terrestre $\mathrm{i}$ cui effetti risultano praticamente apprezzabili ad intervalli di anni o di decenni, quelli cioè di velocità superiore ad 1/10 di mun per decennio e inferiore a quella delle maree terrestri. Essi furono già denominati dall'Issel quali bradisismi. Rimangono esclusi pertanto i sismi propriamente detti e le oscillazioni periodiche dovute all'attrazione luni-solare. Queste due manifestazioni si possono misurare con appropriate apparecchiature.

Problema fondamentale nello studio dei bradisismi è quello della loro misura. Esso appare però indeterminato, nel senso che il piano rispetto al quale dovrebbero essere riferiti gli spostamenti verticali, cioè il livello medio marino varia anch'esso col tempo. Le misure ad esso riferite non possono darci, generalmente, nessuna idea sull'effettivo spostamento verticale del caposaldo. Se un punto della costa mantiene per un lungo intervallo di tempo la stessa quota rispetto ai successivi liv.m. di quel mare, ciò significa solamente che la velocita dello spostamento verticale della costa è uguale a quella del liv.m.m. E ciò costituirà un fatto eccezionale, perché generalmente $i$ due moti non saranno uguali ma diversi, sia di valore che di segrno.

Le misure attualmente eseguite hanno tutte carattere relativo: o si riferiscono ad un punto lontano supposto fisso, oppure si riferiscono al liv.m. di un determinato mare considerato invariabile. In realtà, né l'uno né l'altro sono fermi, ma subiscono col tempo spostamenti verticali continui, senza tener conto dei contemporanei movimenti orizzontali del caposaldo terrestre. Il trascurare questo concetto di relatività dei due moti può portare a gravi errori, come quello di ritenere in fase di sollevamento una costa che in realtà sta abbassandosi.

Il problema ammette però delle soluzioni che possono dare con sufficiente approssimazione $\mathrm{i}$ valori assoluti degli spostamenti rerticali

(*) Comunicazione presentata al Convegno dell'Associazione Geofisica Italiana, tenuto a Roma il 10-11 giugno 1952. 
di caposaldi costieri. La più semplice è quella che permette di determinare per ogni punto terrestre una quota rispetto ad un dato piano fondamentale e le successive sue variazioni. Ciò si può ottenere collegando uno dei due moti relativi, e precisamente quello del liv.m.m., non ad un dato caposaldo terrestre ma alla media delle quote di tutti i caposaldi mareografici. Il che equivale a riferire il liv.m.m. al centro della Terra e supporre trascurabile la variazione del raggio medio terrestre.

2. Il procedimento generale. - Dalle registrazioni mareografiche di stazioni poste lungo le coste di tutti i mari, si determinano i liv.m.m. annui riferendoli a caposaldi locali. Per avere un liv.m. locale meno influenzato da cause di breve periodo, converrà dedurlo da un intervallo più lungo, per es. di 10 anni. Questo liv.m. si attribuisce all'istante centrale dell'intervallo di tempo considerato. Per es. si potrà considerare il liv.m. degli anni 1896-1905 e attribuirlo all'istante 0 del $l^{\circ}$ gennaio 1901. In modo analogo si potrà determinare il liv.m. per il decennio successivo e attribuirlo all'istante 0 del $1^{\circ}$ gennaio 1911. La differenza fra i due liv.m. rappresenterà il valore dello spostamento relativo terra-acqua, per quelle località. Vi saranno delle zone costiere in fase di sollevamento ed altre in fase di abbassamento. Supponendo la variazione del raggio medio terrestre di valore trascurabile rispetto a quello delle variazioni delle singole zone costiere si avrà compensazione fra i movimenti opposti. Facendo allora la media delle variazioni di tutte le regioni costiere della Terra, corrispondenti ad uno stesso decennio, si otterrà un valore nullo, positivo, o negativo secondo che il liv.m. generale è rimasto stazionario, o ha subito un innalzamento, oppure un abbassamento in quel decennio considerato.

Le recenti ricerche sulle variazioni dei liv.m.m. hanno messo in evidenza come $i$ liv.m. di tutti $i$ mari subiscano attualmente un graduale aumento dell'ordine di $1,5 \mathrm{~cm}$ per decennio, rispetto ai caposaldi costieri. Questo aumento avviene in stretta dipendenza con una progressiva fusione delle masse glaciali polari e alpine. Esso non è costante nei successivi intervalli di tempo ma varia principalmente in funzione delle condizioni climatiche generali. Queste dipendono alla loro volta dalla attività periodica e non periodica del Sole.

Nel decennio 1931-40 l'anmento generale è stato di $1,9 \mathrm{~cm}$. A Venezia S. Marco, nello stesso decennio, si è avuto un innalzamento di $4,9 \mathrm{~cm}$. Non potendo ammettere che solo a Venezia per 10 anni consecutivi il liv.m.m. sia stato sempre più alto che nelle regioni vicine, 
si deduce che il suolo, almeno in quella zona ristretta, deve essersi abbassato proprio di $3,0 \mathrm{~cm}$. Il che effettivamente $\dot{e}$ arvenuto, come si è potuto constatare mediante livellazioni geometriche riferite a punti lontani sulla terraferma. Il fenomeno perdura da molti decenni e continua tuttora. Esso potrebbe spiegarsi quale cedimento del suolo dovuto a costipazione del materiale trasportato e depositato dai fumi e dal moto ondoso.

3. Le variazionii decennali generali del livello medio marino. - Per la determinazione dei successivi liv.m. generali sono stati considerati i liv.m. di 110 stazioni mareografiche. Dai liv.m. annui di ciaseuna stazione si dedussero quelli dei decenni 1866-75, ]886-95, ... 1926-35 e 1936-45, che si attribuirono agli istanti 0 del $1^{\circ}$ gennaio J371. 1881, .... 1931 e 194.1. Le differenze fra i liv.m. degli anni centrali 1871 e 1880,1881 e $1890, \ldots . .1931$ e 1940 danno la variazione decennale rispetto al caposaldo locale.

Per dare, nel calcolo della media, lo stesso peso a tutte le regioni della Terra, le stazioni considerate sono state raggruppate in 38 zone costiere, distribuite uniformemente, compatibilmente con la loro disponibiliti, lungo tutti i continenti. Per ciascuna di esse si determinarono le variazioni medie decennali. Si ottennero così $i$ valori che si presentano nella tabella allegata.

Il valore medio per uno stesso decennio, dedotto da quelli di tutte le zone costiere, rappresenta la variazione generale decennale, dà cioè l'innalzamento o l'abbassamento medio di tutti i mari della tabella. II segno + indica innalzamento del livello medio, e il segno - indica l'abbassamento.

La variazione decennale generale non è costante. Il valore medio del settantennio 1871-1940 è di $1,1 \mathrm{~cm}$. È stata massima nell'ultimo decennio (1931-1940). İ stata negativa, si è avuto cioè un abbassamento del liv.m. generale, nell'intervallo 1881-1890.

4. La determinazione dei movimenti verticali costieri. - Riportiamo dalla tabella $i$ valori delle medie generali dei liv.m.m.:

Variazioni decennali generali del liv.m.m. in cm.

(+ innalzamento, - abbassamento)

\begin{tabular}{|c|c|c|c|c|c|c|c|}
\hline $1871-1880$ & $1881-1890$ & $1891-1900$ & $1901-1910$ & $1911-1920$ & $1921-1930$ & $1931-1940$ & $1871-1940$ \\
\hline$+0,9$ & $-1,4$ & $+1,6$ & $+0,2$ & $+0,7$ & $+0,9$ & $+1,9$ & +1.1 \\
\hline
\end{tabular}




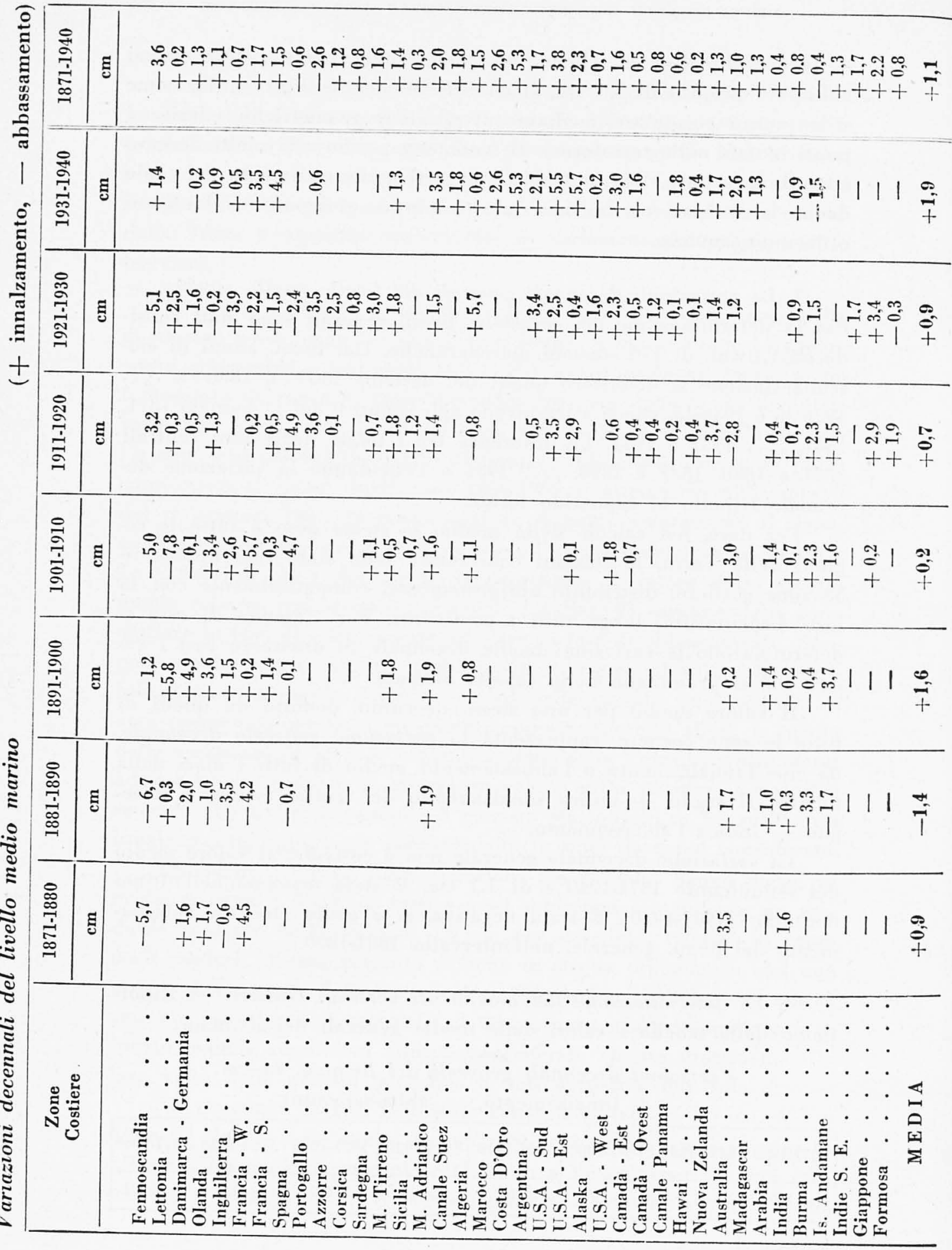


Essi danno dunque la variazione media del liv.m.m. per ciascun decennio del periodo 1871-1940. La differenza tra questi valori e quelli di una data località darà il valore del sollevamento o dell'abbassamento assoluto di quel caposaldo, cioè il valore del suo spostamento verticale indipendentemente dalla variazione del liv.m.m.

5. Precisione delle misure. - La determinazione del bradisismo dipende dunque da due misure: da quella del liv.m. di una stazione e da quella di tutti i mari.

La prima determinazione può essere molto precisa, sia che si usi la media dedotta dalle altezze orarie, sia che si adoperi la media di tutte le alte e le basse maree (in generale i due metodi danno differenze trascurabili, dell'ordine di $1 / 10 \mathrm{di} \mathrm{mm}$ ). La precisione, in ogni caso, è di qualche decimo di $\mathrm{mm}$ e può arrivare ad $1 / 10 \mathrm{di} \mathrm{mm}$ per i livelli medi decennali. Minore precisione si ha nella determinazione del liv.m.m. di tutti i mari. E ciò specialmente per l'arbitrarieta della scelta e del raggruppamento delle stazioni nella determinazione del liv.m.m. generale. $\dot{E}$ evidente che non $\grave{e}$ opportuno fare la semplice media di tutte le stazioni disponibili, conviene invece determinare l'aumento medio per ogni regione costiera e fare la media di questi valori, scelti anch'essi in modo conveniente. Riteniamo che un lavoro accurato possa dare l'aumento medio decennale di tutti i mari con la precisione di $1 / 2 \mathrm{~mm}$. Per lo spostamento verticale assoluto dei capisaldi mareografici la precisione risulta, per quanto si disse, di qualche decimo di $\mathrm{mm}$.

6. Considerazioni generali. - Si notino l'alto grado di precisione che si può ragrgiungere con questo procedimento nella determinazione degrli spostamenti verticali costieri e l'importanza che può assumere una continua e regolare registrazione mareografica sia nella ricerca oceanografica che in quella geofisica.

Quale immediata applicazione pratica del procedimento indicato sono stati determinati gli attuali morimenti verticali delle coste continentali. I risultati di questa elaborazione figurano separatamente nella relazione che segue a questa nota.

$$
\text { Trieste - Istituto Talassografico - Giugno } 1952 .
$$




\section{RIASSUNTO}

Si indica un procedimento che consente di misurare $i$ lenti movimenti verticali della superficie terrestre mediante determinazioni ma. reografiche di livelli medi marini. Esso vale tanto per zone ristrette che vaste. Si applica direttamente alle regioni costiere e mediante una livellazione di precisione alle zone interne. Esso si basa sulla determinazione della differenza fra le velocità del movimento medio verticale del livello di tutti i mari e quello di una determinata stazione. Tale differenza dà lo spostamento verticale assoluto della costa nell'intervallo considerato. Dai dati di tutte le stazioni mareografiche della Terra, raggruppati per decenni e per regioni costiere, si determinano, per il periodo 1871-1940, le variazioni decennali medie sia delle regioni costiere che quelle generali di tutti $i$ mari. In questo periodo di tempo l'aumento medio del livello marino è stato di $1,1 \mathrm{~cm}$ per decennio. Il metodo consente di misurare spostamenti verticali dci caposaldi costieri con una precisione che è dell'ordine di pochi decimi di mm per decennio.

\section{SUMM-ARY}

A procedure is demonstrated that permits the calculation of slow vertical movements of the earth's surface by means of marinografic determinations of the mean ocean levels. This procedure is valid for large zones as vell as for those more restricted. Results obtained from the coastal regions can be applied to the inland regions by means of a precise correlation. This method is based on the determination of the difference between the velocity of the mean vertical movement of all the seas and that of a designated station. Such a difference gives the absolute vertical displacement of the coast in the region considered. From the data from all the marine stations of the Earth, grouped, in ten-year intervals, and further by coastal region, the mean ten-yearly variation, of some coastal regions from the mean of all the oceans for the period 1871-1940, has been calculated.

In this period of time the mean increase of the level of the sea has been $1.1 \mathrm{~cm}$ per ten years. The method permits the measurement of vertical displacements of the coastal level with a precision that is on the order of a few tenths of a millimeter for a ten-year interval. 


\section{BIBLIOGRAFIA}

Assoc. Ocean. Phys, Monthly and annual mean heights of sea-level. Union Geod. Geoph. Intern, Publ. Scient. n. 5 (Liverpool, 1940) e n. 10 (Oslo, 1950).

PoLLI S, Livelli medi, capisaldi di livellazione e ampiezze delle maree nel porto di Trieste. Mem. 253, Comit. Talassogr. It. del C.N.R., 1938.

PolxI S, Il graduale aumento del livello marino determinato per 30 porti del Mediterraneo. Boll. Soc. Adriatica di Sc. Nat, Trieste, vol. XLII, 1946.

Polli S., Il graduale aumento del livello del mare a Venezia, Trieste e Pola. Geofisica pura e applicata, Milano, vol. IX, fase. 1-2, 1946.

Polli S, Livello medio del mare nella livellazione di precisione. Boll. Soc. Adriatica di Sc. Nat., XLII (1946), 52-56, Trieste.

Poln S., Gli attuali movintenti verticali delle coste italiane. Tecnica Italiana, Trieste, N.S, 4, 1947.

Poln S., Registrazione dei bradisismi costieri. Geofisica para e applicata, Milano, vol. XII, fasc. $34,1948$. 